\title{
Performance of Fractionally Spaced MLSE in OOK and PAM4 Bandwidth Limited Optical Systems
}

\author{
Cristian Prodaniuc, Nebojsa Stojanovic, Member, IEEE, and Zhang Qiang
}

\begin{abstract}
We analyze the performance of fractionally spaced maximum likelihood sequence estimation (MLSE) equalizers in OOK and PAM4 optical systems using optical and electrical components with cut-off frequencies less than the baud rate. It has been demonstrated that signals suffering from optical and electrical impairments can be efficiently equalized in cheap direct-detection optical receivers using MLSE equalizers with one or two samples, depending on the extent of bandwidth limitations.
\end{abstract}

Keywords - Maximum likelihood sequence estimation, chromatic dispersion, bandlimited systems, direct detection, digital receiver.

\section{INTRODUCTION}

$\mathrm{C}$ OHERENT optical systems using both polarizations and high-level modulation formats enable data transmission at much higher bit rates compared with noncoherent systems [1]. Most noncoherent systems are based on intensity modulation with direct detection (IM$\mathrm{DD}$ ) also known as on/off keying (OOK) and non-return to zero (NRZ). These systems suffer from chromatic dispersion (CD) and polarization mode dispersion (PMD), and their deployment was limited to rates up to $10-\mathrm{Gb} / \mathrm{s}$. Transponders for $40-\mathrm{Gb} / \mathrm{s}$ systems use slightly more complex modulation, such as optical duobinary (ODB) and differential phase shift keying (DPSK), and increasingly crowding out $10-\mathrm{Gb} / \mathrm{s}$ systems due to increased traffic demands. Further improvements have been achieved by noncoherent $40-\mathrm{Gb} / \mathrm{s}$ DQPSK transponders that also suffer from fiber impairments and require expensive external PMD compensators. Most commercial optical links contain dispersion compensation fibers (DCF) and CD has become uncritical for noncoherent systems.

Direct detection systems are very attractive because of price, power consumption, size, and so on. In IM-DD 10$\mathrm{Gb} / \mathrm{s}$ systems, the received signal can be equalized by several techniques such as feed-forward (FFE), decision feedback (DFE), and MLSE equalizers. FFE equalizers are the least efficient solution while DFE can extend optical

Paper received April 2, 2015; revised September 18, 2015; accepted September 20, 2015. Date of publication November 15, 2015. The associate editor coordinating the review of this manuscript and approving it for publication was Prof. Dejan Gvozdić.

This paper is a revised and expanded version of the paper presented at the 22th Telecommunications Forum TELFOR 2014.

The authors are with Huawei Technologies Duesseldorf GmbH, Riesstrasse 25, 80992, Munich, Germany. (e-mail: cristian.prodaniuc@huawei.com). reach but suffers from error multiplication at a low optical signal-to-noise ratio (OSNR). Additionally, DFE equalizers experience implementation problems at high bit rates. The MLSE technique is the best one, enabling the longest reach and best performance at the price of very complex digital signal processing (DSP). However, IC technology advances make complex MLSE equalizers feasible. For example, the power consumption of a 64state MLSE equalizer realized in 28nm technology for 28 $\mathrm{Gb} / \mathrm{s}$ binary systems is approximately $1.5 \mathrm{~W}$.

The Viterbi algorithm significantly reduces finding the most likely transmitted data sequence and enables the realization of practical MLSE decoders [2]. Besides their usage in convolutional decoders and many other applications, MLSE equalizers can also be used in direct detection optical receivers for compensating intersymbol interference caused by CD and PMD [3-6]. Commercial $10-\mathrm{Gb} / \mathrm{s}$ MLSE receivers and DSP chips including the Viterbi decoder with 4,8 or 16 states have been available for many years now. In some realizations, MLSE and FFE equalizers are combined to decrease the total complexity.

Dual polarization $100-\mathrm{Gb} / \mathrm{s}$ coherent systems based on QPSK modulation formats are already standardized and deployed all over the world to solve network bandwidth growth driven by cloud, mobile, and video. Coherent receivers can efficiently compensate $\mathrm{CD}, \mathrm{PMD}$, nonlinear effects, and so on. They require very expensive modulators, demodulators, and power-hungry DSP. New optical networks are not supposed to deploy DCF anymore because coherent optical systems are no longer limited by $\mathrm{CD}$. The price of coherent transponders is still very high. That limits their usage to long-haul applications. In shorter optical links, coherent technology is very expensive, and less expensive solutions should be developed to enable $100-\mathrm{Gb} / \mathrm{s}$ traffic. Therefore, some cheap solutions have been proposed and already commercialized [7]. The solution in [7] is based on IM-DD systems using 10-G components to carry a $28-\mathrm{Gb} / \mathrm{s}$ signal. The transmitted signal suffers from ISI caused by the narrow bandwidth of $10-\mathrm{G}$ components and optical impairments. ISI is efficiently compensated by MLSE equalization. In the last year research on IM-DD systems is changing its focus from OOK to pulse amplitude modulation 4 (PAM4) $[8,9]$ and duobinary [10] modulation.

MLSE complexity in solutions based on cheap narrowband components is discussed in this paper. We analyze MLSE efficiency for OOK and PAM4 modulation formats. We compare performance in receivers using one 


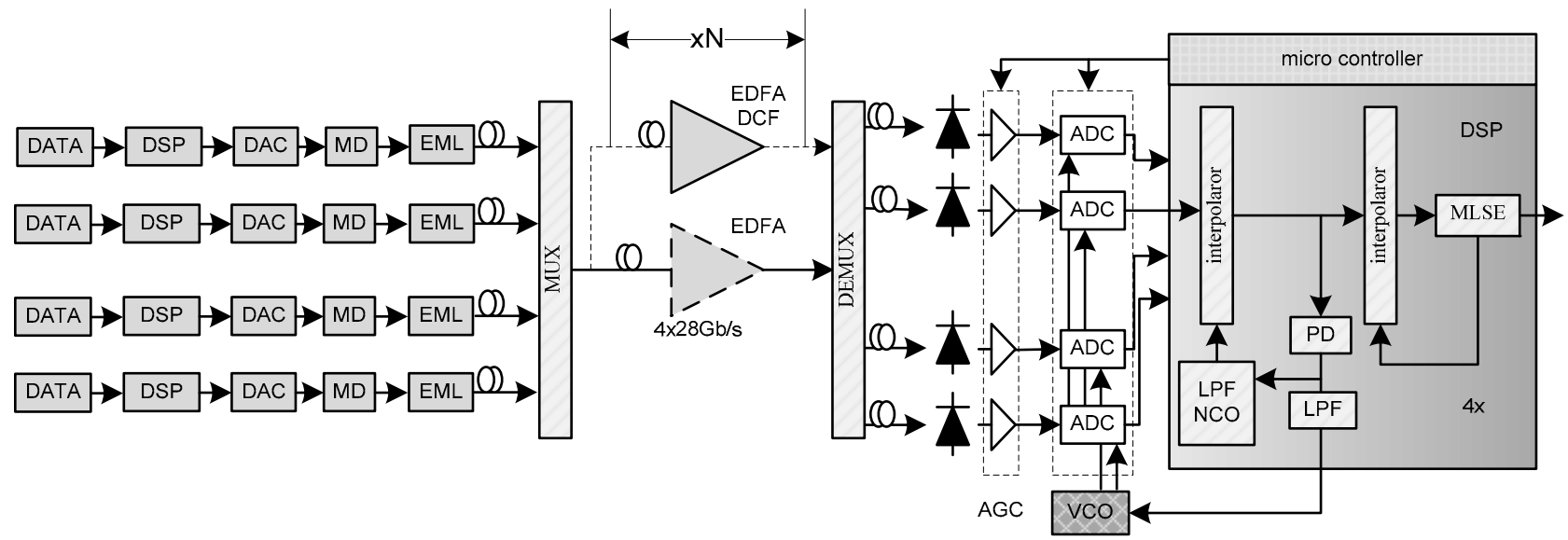

Fig. 1. 100-G transmission system.
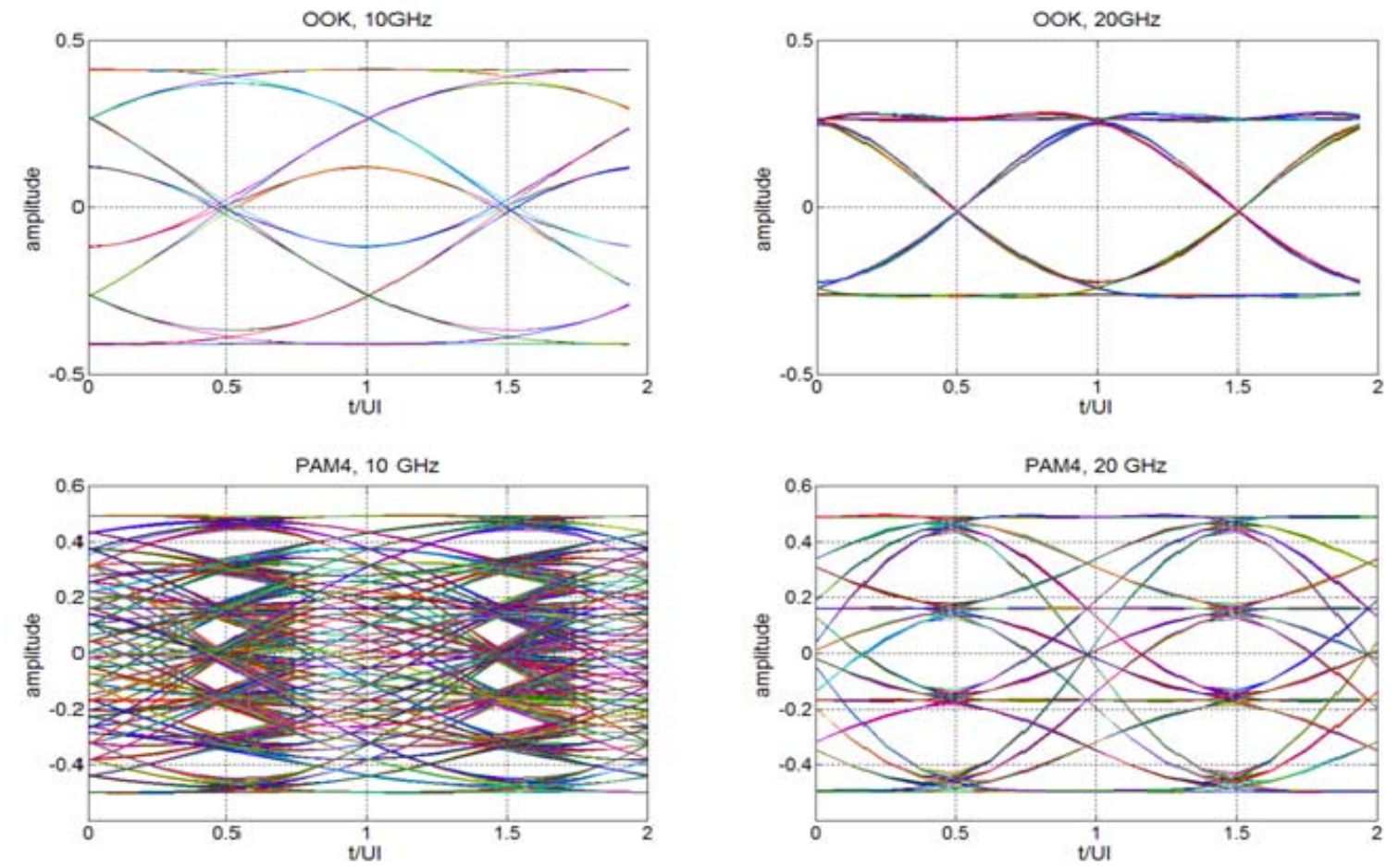

Fig. 2. Eye diagrams of 10 and $20 \mathrm{GHz} \mathrm{Rx} / \mathrm{Tx}$ in OOK and PAM4 28-Gbaud/s systems.

and two samples per symbol to be able to decide on the required number of MLSE samples per symbol because the complexity can be greatly reduced when only one sample is used.

\section{MLSE EQUALIZATION IN OPTICAL RECEIVERS}

The block diagram of a $100-\mathrm{G}$ transmission system using four wavelengths each carrying a $28 \mathrm{~Gb} / \mathrm{s}$ signal is presented in Fig. 1. The binary signal is first processed by DSP at the transmitter side to compensate for the narrow bandwidth of the transmission system. Cheap 10-G components have bandwidths less than $10-\mathrm{GHz}$ and the whole system may end up with less than $6-\mathrm{GHz}$ bandwidth. Therefore, Tx DSP is used to increase the bandwidth of transmission system and improves BER performance by several $\mathrm{dB}$ or $\mathrm{dBm}$. Digital-to-analog converters (DAC) are required to generate analog signals that are amplified by modulator drivers (MD). Signals after MDs directly modulate (intensity modulation) laser signals integrated in electro absorption modulators (EAM). Laser and EAM are often integrated in an externally modulated laser (EML). Four signals of $28 \mathrm{~Gb} / \mathrm{s}$ data are multiplexed into a single-mode fiber. There are two transmission scenarios, point-to-point and transmission via several optical spans. Each span includes a single mode fiber and an erbium-doped fiber amplifier (EDFA). In the first scenario, one EDFA may be required in longer links when the input optical power is below a certain threshold (related to receiver sensitivity). At the receiver side, the optical signal is demultiplexed and directly converted into an electrical signal. If the optical front end does not include an automatic gain control (AGC), which is extremely important for proper MLSE equalization and prevents signal clipping, an AGC block has to be added per each data line. After an analog-to-digital (ADC) conversion, the quantized signal is processed by MLSE. $\mathrm{AGC}$ and $\mathrm{ADC}$ can be controlled via a micro-controller that gets the control information from the DSP block. Timing information is extracted by a phase detector (PD) and filtered by low-pass filters (LPF). This information is used in an external PLL to control the ADC sampling phase and frequency via a voltage-controlled oscillator 
(VCO), and in an inner loop via a numerically controlled oscillator (NCO) for tracking fast jitter variations. To optimize MLSE performance, an additional interpolator is required in front of the MLSE block.

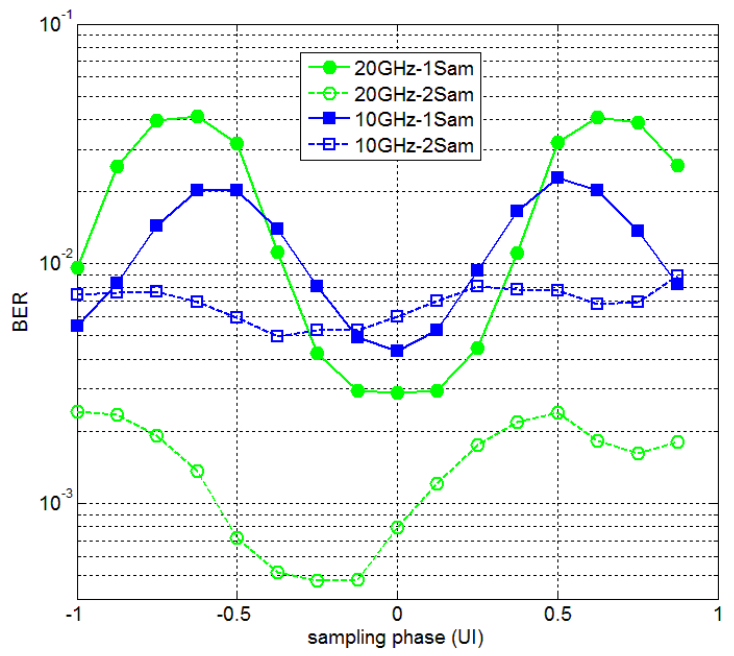

Fig. 3. Performance of 16-state MLSE at CD of 700 $\mathrm{ps} / \mathrm{nm}$.

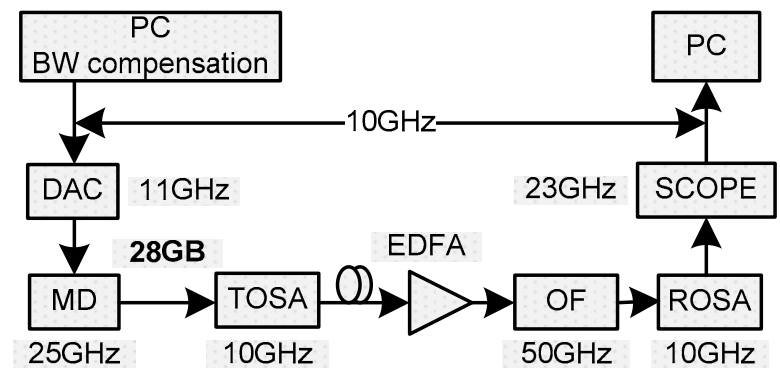

Fig. 4. OOK experimental setup.

The MLSE equalizer can use different branch metrics: Euclidian distance, metrics derived from histograms, metrics derived from supposed probability density functions, among others. Histogram-based MLSE equalizers provide the best performance at the price of very high complexity when ADC resolution is high. The simplest solution employs Euclidian distance and metrics that are in fact the mean values of estimated histograms. The amount of ISI that can be compensated depends on the number of states implemented in the MLSE equalizer. Increasing memory length by one symbol doubles the complexity of binary MLSE or it quadruples the complexity in case PAM4 is used. This complexity cannot be easily reduced without penalties. In general, fractionally spaced MLSE depends less on a sampling phase and provides a better performance than MLSE relying on only one sample per symbol.

The system shown in Fig. 1 is simulated with the following parameters: EML extinction ratio of 11.5 and chirp of $-0.1, \mathrm{Rx}$ and $\mathrm{Tx}$ bandwidths were set to 10 and 20 $\mathrm{GHz}$ (each of them), and OSNR was $200 \mathrm{~dB}$. All simulations are carried out in MATLAB. The corresponding $\mathrm{Rx}$ electrical eye diagrams over 2 unit intervals (UI) are presented in Fig. 2. The 10-GHz system generates large ISI that must be compensated as BER performance is dramatically reduced after simple hard decision data recovery. In the next two chapters we will analyze the performance of fractionally spaced MLSE, first for OOK modulation and then for PAM4 modulation. For both cases we include simulation and experimental results.

\section{MLSE PERFORMANCE WITH ONE AND TWO SAMPLES PER SYMBOL FOR OOK MODULATION}

To evaluate MLSE performance with one and two samples per symbol, for an OOK transmission, we simulated a 16-state MLSE and set CD to $700 \mathrm{ps} / \mathrm{nm}$ and OSNR to $19 \mathrm{~dB}$. Transmitter and receiver bandwidths were set to 10 and $20 \mathrm{GHz}$. BER performances over two symbols are presented in Fig. 3. In the case of $20 \mathrm{GHz}$ bandwidth, at the best sampling point, two samples per symbol achieved about six times better performance than a single sample MLSE. Two samples per symbol MLSE is also sensitive to sampling phase because the 16-state MLSE is at the limit for the simulated CD value. The bandwidth decreasing by a factor of 2 leads to a much worse performance of a two-sample MLSE while a single-
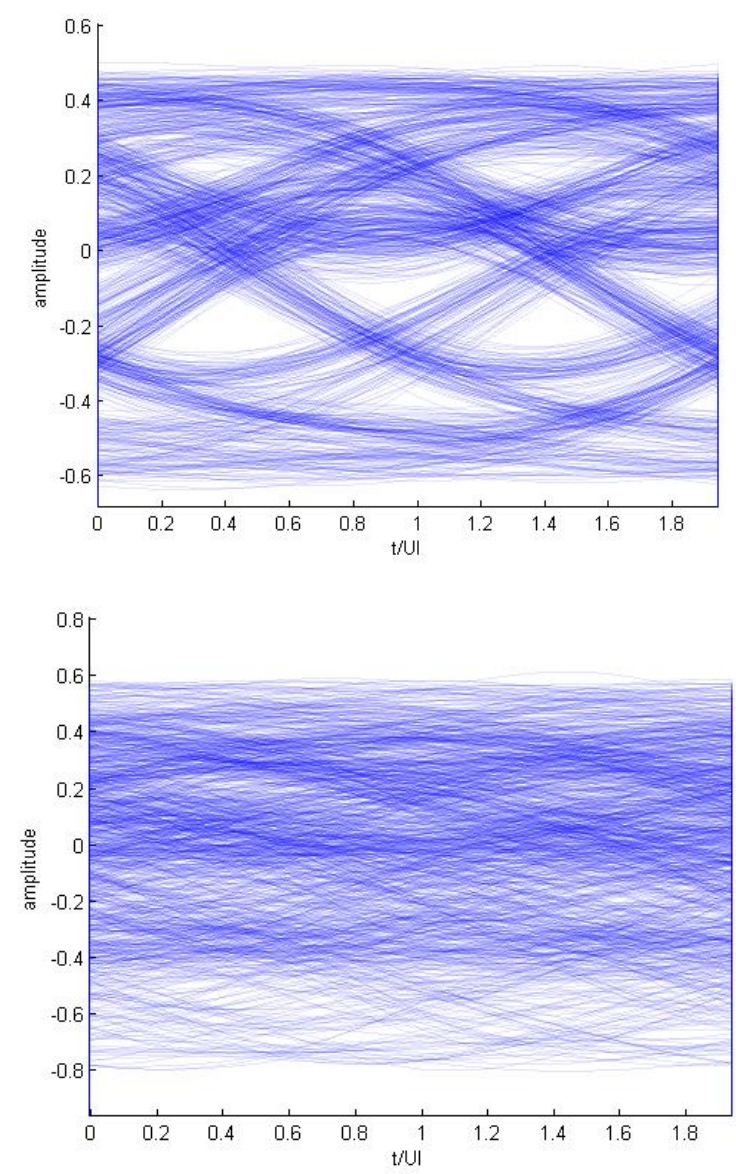

Fig. 5. Eye diagrams in experiments at received optical power of $-10 \mathrm{dBm}$ for $\mathrm{B} 2 \mathrm{~B}$ and after $80 \mathrm{~km}$ for OOK.

sample MLSE did not suffer significantly. The singlesample MLSE performs better at the best sampling point.

The next step was to use experimental data to check the simulation results. We used the experimental setup presented in Fig. 4. At the transmitter side, a pseudorandom bit sequence is used to generate the offline data using Matlab. Pre-distortion of the signal is performed to compensate for the limited bandwidth of the transmitter. 


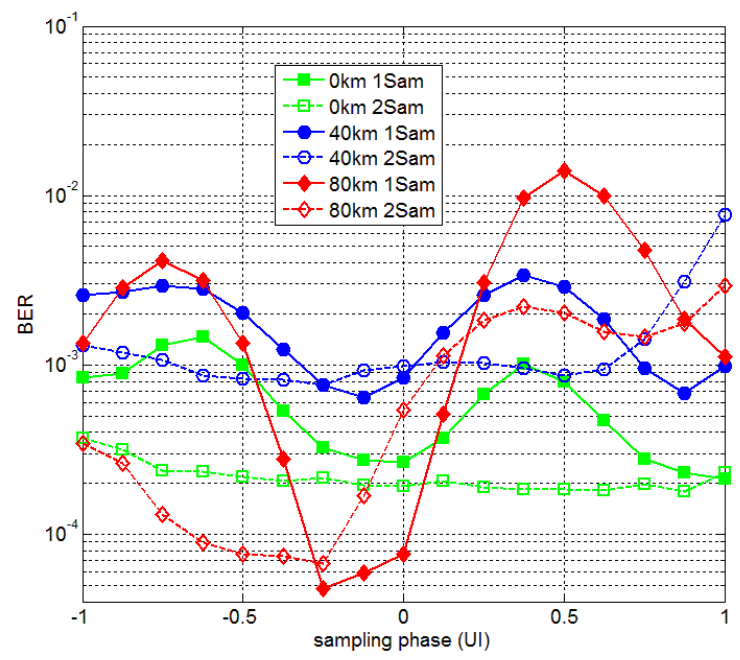

Fig. 6. BER variations in experiments after 0,40 , and $80 \mathrm{~km}$ links.

Then, the pre-distorted data are up-sampled to 2 Sam/Symbol and are loaded to a DAC with an 8-bit resolution and 11-GHz 3-dB analog bandwidth. The DAC operates at $56 \mathrm{GSam} / \mathrm{s}$ leading to the generation of $28 \mathrm{~Gb} / \mathrm{s}$ NRZ-OOK electrical signal at its differential outputs which are fed into a $25-\mathrm{GHz} 3-\mathrm{dB}$ bandwidth RF driver to amplify and to modulate a $10-\mathrm{GHz}$ transmitter optical subassembly (TOSA). The TOSA consists of a distributed feedback laser (DFB) and EAM modulator integrated in the same module. Next, the $28-\mathrm{Gb} / \mathrm{s}$ NRZ-OOK optical signal is launched over a single-mode fiber, amplified by an EDFA, and filtered through a $50-\mathrm{GHz}$ optical filter $(\mathrm{OF})$. At the receiver side, the received optical power is changed using a variable optical attenuator (not presented in the figure) and the signal is finally detected on a 10$\mathrm{GHz}$ receiver optical sub-assembly (ROSA). The ROSA consists of a $p$-i-n photodiode integrated together with a trans-impedance amplifier (TIA). The received electrical eye diagrams in a back-to-back case (B2B) and after 80 $\mathrm{km}$ for the received optical power of $\mathrm{Pin}=-10 \mathrm{dBm}$ are shown in Fig. 5. After comparing the experimental B2B eye diagram with that in simulation (see Fig. 2 left), one can conclude that electrical noise at both sides (Tx and Rx) is quite large. Additionally, ROSA introduced very strong signal clipping at high signal values. The $80-\mathrm{km}$ eye diagram is completely closed, indicating that without an enhanced equalization signal, recovery is impossible.

We estimated MLSE performance with one and two samples at BER between $10^{-3}$ and $10^{-4}$. Links of 0,40 , and $80 \mathrm{~km}$ at input optical power of $-18,-16$, and $-14 \mathrm{dBm}$, respectively, were analyzed. A 16-state MLSE was used in the first two cases while a 64-state MLSE was simulated in the $80-\mathrm{km}$ link. BER variations over two UI are presented in Fig. 6. One and two samples MLSE equalizers provide a similar performance. In the B2B case and 40-km link, two samples are more resilient to the sampling phase while this advantage disappears at very high CD value after the 80 $\mathrm{km}$ link. Note that the single-sample MLSE equalizer has a slightly better performance at the best sampling phase compared to the two-sample MLSE equalizer. In general, based on these results, we have realized that two samples

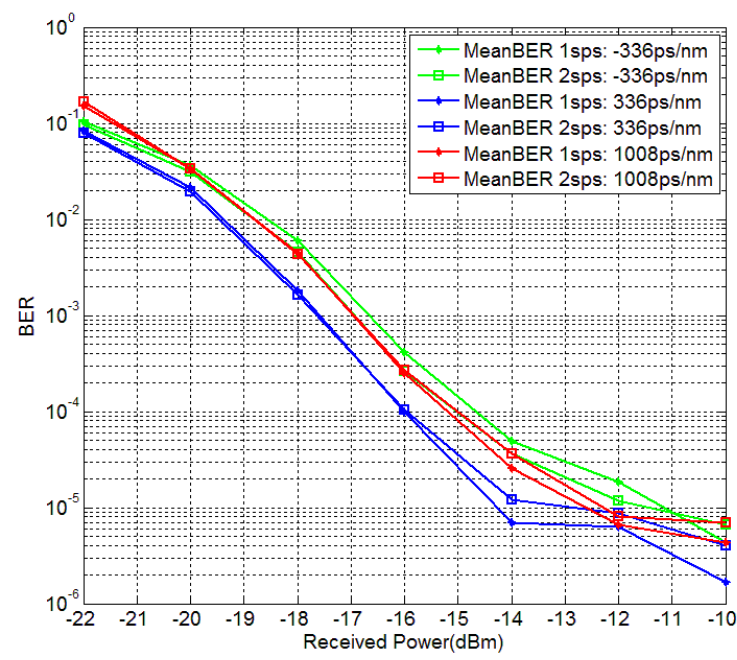

Fig. 7. Minimum BER versus received optical power in experiments for three different optical links.

per symbol are not required in strongly bandlimited systems. This agrees with the simulation results presented in Fig. 3 (see $10 \mathrm{GHz}$ lines). One of the most critical points is to find the best sampling phase. Sampling phase variations introduce more serious penalties in singlesample MLSE. We analyzed the BER minima and maxima closest to the sampling phase 0 . In the $80-\mathrm{km}$ case, at $0.5 \mathrm{UI}$ phase, BER is greater than $10^{-2}$ while the minimum BER of $5 \times 10^{-5}$ is achieved at a -0.25 phase. For the same link, two samples yield a BER minimum equal to $7 \times 10^{-5}$ at a -0.25 while the maximum BER of 0.002 is located at a 0.375 phase. In this region, one sample experienced onedecade higher BER variations than a two-sample MLSE.

In the next experiment, we used three links with $\mathrm{CD}$ values of $-336,336$, and $1008 \mathrm{ps} / \mathrm{nm}$. Negative dispersion was achieved by using a DCF fiber with negative dispersion. Optical power was varied from -10 to -22 $\mathrm{dBm}$. The 64-state MLSE equalizer was used in 1008 $\mathrm{ps} / \mathrm{nm}$ link while in the other two cases, a 16-state MLSE was simulated. Minimum BER estimated at the best sampling phase versus input optical power for one- and two-sample MLSE is shown in Fig. 7. BER is averaged over three data sets to get more accurate results. The two samples per symbol MLSE performs slightly better at negative dispersion. In the other two channels, there was no significant difference. This is an additional proof that a much more complex fractionally spaced MLSE is not required in the transmission scenario utilizing cheap optical components with cut-off less than the Nyquist frequency. Note that in the last results, we have used the proprietary clock recovery with a loop bandwidth of 2 $\mathrm{MHz}$ (second-order phase locked loop) to get a more realistic performance. Clock recovery is one of the most difficult problems to solve in bandlimited systems that additionally suffer from serious ISI caused by CD.

\section{MLSE PERFORMANCE WITH ONE AND TWO SAMPLES PER SYMBOL FOR PAM4 MODULATION}

In this chapter we analyze the performance of MLSE with one and two samples per symbol, when PAM4 is employed as a modulation format. Compared with OOK, 
PAM4 requires higher OSNR values in order to achieve the same BER. However, at the same bit rate, PAM4 requires half the bandwidth and has better $\mathrm{CD}$ tolerance, as can be seen in Fig. 8, where no bandwidth limitations were

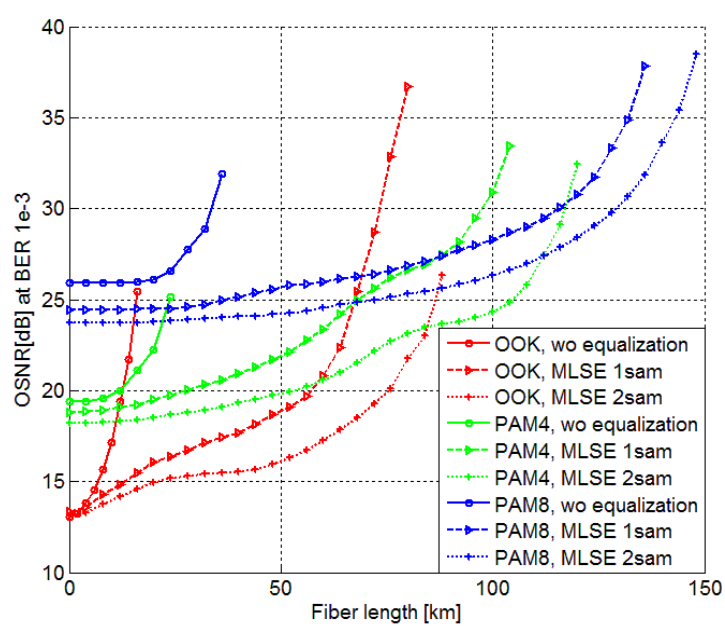

Fig. 8. Transmission reach for OOK(red), PAM4 (green) and PAM8 (blue) when using 64 states MLSE.

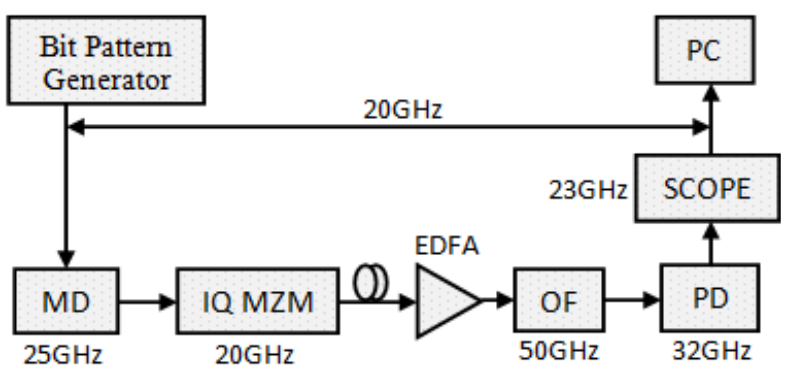

Fig. 9. PAM4 experimental setup.

imposed and a 64-state MLSE was used for equalization. OOK allows us to reach around $80 \mathrm{~km}$ fiber lengths while maintaining the BER below $10^{-3}$. For the same BER threshold PAM4 can be used for fiber lengths of up to $120 \mathrm{~km}$.

To assess the performance of one and two samples per symbol MLSE in the context of PAM4 modulation, we simulated receiver and transmitter bandwidths of $10 \mathrm{GHz}$ and $20 \mathrm{GHz}$ and set the OSNR at $24 \mathrm{~dB}$ and the $\mathrm{CD}$ at 170 $\mathrm{ps} / \mathrm{nm}$. The baud rate is $28 \mathrm{Gbaud}$, resulting in a bit rate of 56 Gbps. Fig. 11 shows the simulation results with these parameters. As in the case of OOK modulation, a 16-state MLSE was used. It must be mentioned that for PAM4 the complexity of MLSE grows faster with the increase of memory bits. In the case of $20 \mathrm{GHz}$ bandwidth the fractionally spaced MLSE performs two times better than its single sample counterpart, achieving a minimum BER of $4 \times 10^{-4}$ and the single sample MLSE a minimum BER of $9 \times 10^{-4}$. When limiting the bandwidth to $10 \mathrm{GHz}$ the performance stays the same and even improves somewhat for one sample per symbol MLSE. This can be attributed to the fact that a lower bandwidth also limits some of the noise while the MLSE can compensate almost entirely for the lost signal bandwidth.

As in the case of OOK, also for PAM4 we setup an experiment to test the performance of fractionally spaced
MLSE. The block diagram of this setup can be seen in Fig. 9. Two pseudo-random bit sequences are generated and loaded into the bit pattern generator (BPG). The BPG then outputs two OOK signals which are fed to a couple of 25 $\mathrm{GHz}$ bandwidth drivers. Next the amplified signals are sent to a $20 \mathrm{GHz}$ bandwidth IQ Mach-Zehnder modulator (MZM) which will modulate a continuous wave laser (CWL) and generate at its output the optical PAM4 signal. The optical signal is then launched over single-mode fibers of lengths 0,4 and $8 \mathrm{~km}$. The optical signal is amplified with the help of an EDFA and filtered by a $50 \mathrm{GHz}$ bandwidth OF. The optical eye diagrams after EDFA for 0 and $8 \mathrm{~km}$ can be seen in Fig. 10. It shows that for $8 \mathrm{~km}$ the signal is already heavily distorted, more than can be attributed to $\mathrm{CD}$ alone. This is due to the chirp introduced by the IQ MZM when generating PAM4 signal [11]. The receiver consists of a $\mathrm{p}-\mathrm{i}-\mathrm{n}$ photodiode. After the receiver a real-time oscilloscope is used to capture the data at a

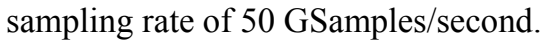
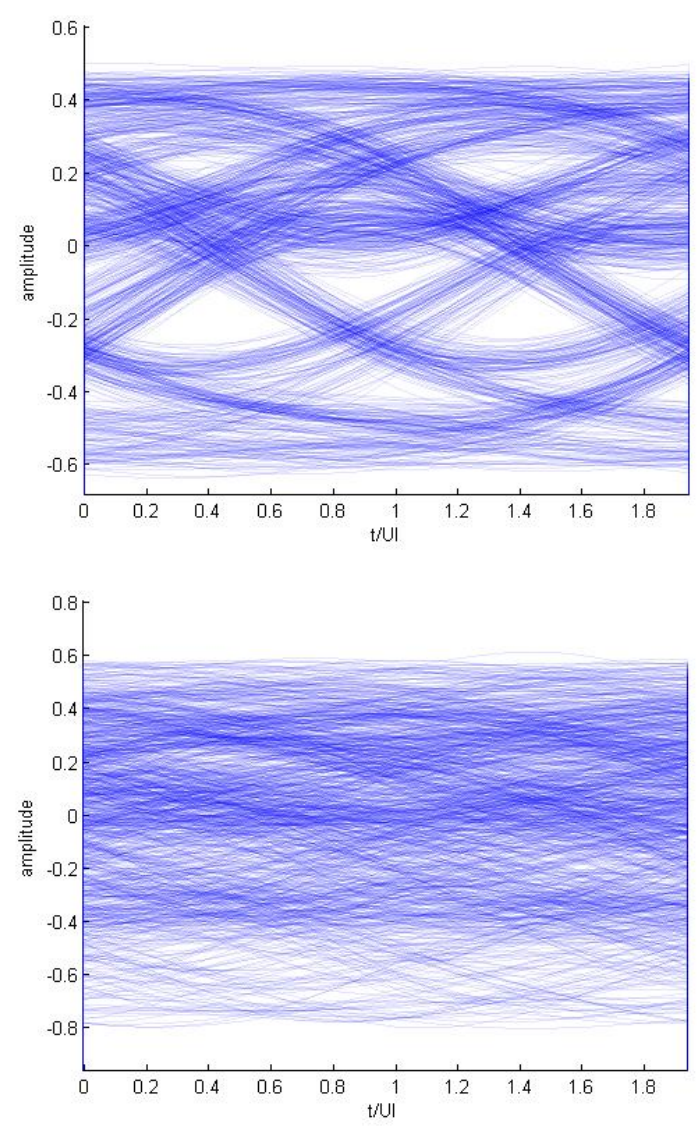

Fig. 10. Eye diagrams in experiments at received optical power of $5 \mathrm{dBm}$ for B2B and after $8 \mathrm{~km}$ for PAM4.

We evaluated the performance of one and two samples MLSE equalizers for links of 0,4 and $8 \mathrm{~km}$. For lengths of 0 and $4 \mathrm{~km}$ a 16-state MLSE was employed, while for 8 $\mathrm{km} 64$ states were needed. The achieved BER for this experimental setup after MLSE equalization can be seen in Fig. 12. The missing points in this figure represent a BER of 0 . However, due to the limited amount of offline data that have been processed $\left(2^{19}\right.$ symbols for each BER point), values below $10^{-5}$ are not very precise and a BER 
of 0 should be interpreted as a BER below $10^{-5}$. In the BTB case both the single sample and the fractionally spaced MLSE are able to eliminate all errors, the latter option being more stable. For the 4 and $8 \mathrm{~km}$ transmissions two samples perform much better. While with an one-sample MLSE we have achieved a BER of $5 \times 10^{-5}$ and $4 \times 10^{-4}$ for the 4 and $8 \mathrm{~km}$ links, respectively, the two-sample equalizer will correct all errors when transmitting over a $4 \mathrm{~km}$ link and lower the BER to $10^{-5}$ for $8 \mathrm{~km}$. For both cases there is a significant improvement of over a decade (more than 10 times) in BER. It must be noted that the $3 \mathrm{~dB}$ bandwidth of the whole system in the PAM4 experiment is around $20 \mathrm{GHz}$, such that the bandwidth limitations are less severe than in the case of the OOK experiment. Simulation results from Fig. 8 suggest that when bandwidth is not limited the MLSE equalizer with two samples will perform better.

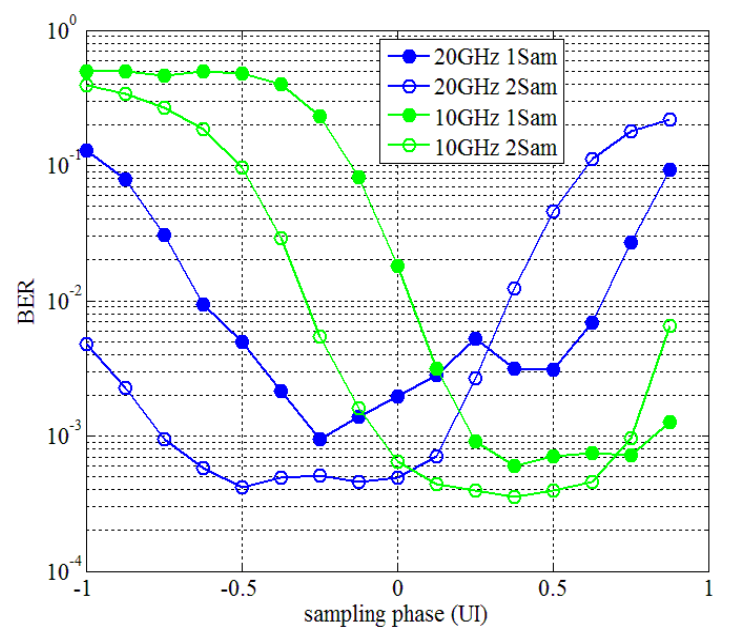

Fig. 11. Performance of 16-state MLSE at CD of 170 $\mathrm{ps} / \mathrm{nm}$ and PAM4 modulation.

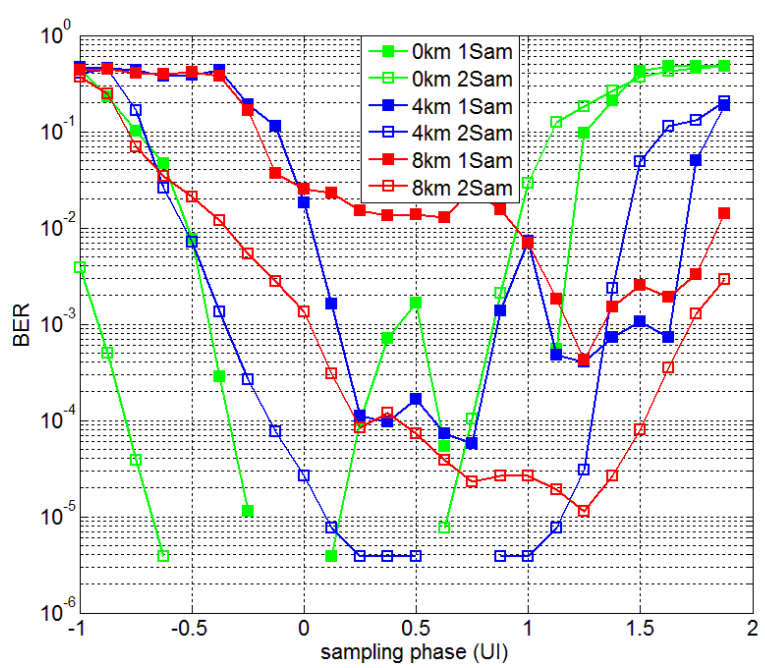

Fig. 12. BER variations in PAM4 experiments after 0 , 4 , and $8 \mathrm{~km}$ links.

\section{CONCLUSION}

We have demonstrated, both in simulations and experiments and for OOK and PAM4 modulation techniques, the capabilities of fractionally spaced MLSE equalization in comparison with its single sample counterpart. Optical and electrical components for 10-G IM-DD systems can be used to carry a $28-\mathrm{Gb} / \mathrm{s}$ signal that is equalized by using the MLSE technique. For this purpose a single-sample MLSE gives approximately the same results as the fractionally spaced MLSE. Compared with two samples, a single sample decreases the ASIC complexity by up to $30 \%$, depending on the level of parallelization and MLSE parameters selection, among others. If better components are used, such that the bandwidth is not too limited, then two-sample MLSE equalization will bring a significant gain in terms of BER which can overcome the cost of the $30 \%$ increase in complexity.

\section{REFERENCES}

[1] C.R.S. Fludger, T. Duthel, D. van den Borne, C. Schulien, E.-D. Schmidt, T. Wuth, J. Geyer, E. De Man, G.-D. Khoe, and H. de Waardt, "Coherent equalization and POLMUX-RZ-DQPSK for robust 100-GE transmission," J. Lightw. Technol., vol. 26, pp. 64 72, Jan. 2008.

[2] G.D. Forney, Jr., "The Viterbi algorithm," Proc. IEEE, vol. 61, pp. 268-278, Mar. 1973.

[3] H.F. Hauntein, K. Sticht, A. Dittrich, W. Sauer-Greff, and R. Urbansky, "Design of near optimum electrical equalizers for optical transmission in the presence of PMD," in Proc. OFC, Anaheim, Mar. 2001, pp.WAA4-1-WAA4-3.

[4] J.P. Elbers, H. Wernz, H. Griesser, C. Glingener, A. Faerbert, S. Langenbach, N. Stojanovic, C. Dorschky, T. Kupfer, and C. Schulien, "Measurement of the dispersion tolerance of optical duobinary with an MLSE-receiver at $10.7 \mathrm{~Gb} / \mathrm{s}$," in Proc. OFC, Anaheim, Mar. 2005, paper OthJ4.

[5] O.E. Agazzi, M.R. Hueda, H.S. Carrer, and D.E. Crivelly, "Maximum-likelihood sequence estimation in dispersive optical channels," J. Lightwave Technol. Vol. 23, pp. 749-763, Feb. 2005.

[6] H.M. Bae, J.B. Ashbrook, J. Park, N.R. Shanbhag, A.C. Singer, and S. Chopra, "An MLSE Receiver for Electronic Dispersion Compensation of OC-192 Fiber Links," IEEE J. Solid-State Circuits, vol. 41, pp. 2541-2554, Dec. 2006.

[7] A. Gorshtein, O. Levy, G. Katz, and D. Sadot, "Low cost 112 G direct detection metro transmission system with reduced bandwidth (10G) components and MLSE compensation," In Proc. SPPCoM, Toronto, June 2011, paper SPWC4

[8] J. Man, W. Chen, X. Song, and L. Zeng, "A Low-Cost 100GE Optical Transceiver Module for 2km SMF Interconnect with PAM4 Modulation,” OFC 2014, March 2014, paper M2E.7.

[9] J. Man, W. Chen, X. Song, and L. Zeng, "A Low-Cost 4x25Gbps PAM4 Module for Short-Reach Optical Interconnection," Optical Interconnects Conference 2014, paper 6886112.

[10] L. F. Suhr, J.J. Vegas Olmos, B. Mao, X. Xu, G. N. Liu, I. Tafur Monroy, "112-Gbit/s x 4-Lane Duobinary-4-PAM for 400GBase," ECOC 2014, September 2014, paper Tu.4.3.2.

[11] A. Dochhan, M. O. Al-Dwairi, and W. Rosenkranz, "Optimization of Cost Efficient Multilevel-ASK Modulation Formats under the Constraint of Chromatic Dispersion," OFC/NFOEC 2010, March 2010, paper EMJ7 